\title{
INVESTIGATION OF INTERFACE STATES IN Si/NAOS-Si0/2/HFO STRUCTURES USING COMPLETE ACOUSTIC SPECTROSCOPY
}

The set of MOS structures formed on n-type Si substrate with (NAOS)-SiO $2_{2} / \mathrm{HfO}_{2}$ gate dielectric layers was prepared and annealed in $\mathrm{N}_{2}$ atmosphere at various temperatures to stabilize the structure and to decrease the interface states density. Two Acoustic DLTS techniques using both surface (SAW) and longitudinal (LAW) acoustic waves including acoustoelectric response signal versus gate voltage dependence ( $U_{a c}-U_{g}$ characteristics) were used to characterize the interface states and the role of annealing treatment. The main interface deep centers with activation energies about 0.30 and $0.20 \mathrm{eV}$, typical for dangling bonds were observed as well as a particular influence of annealing treatment on the interface states. The obtained results are analyzed, discussed and mutually compared.

Keywords: Acoustic DLTS, MOS structures, $\mathrm{NAOS} \mathrm{SiO}_{2} / \mathrm{HfO}_{2}$ oxide layer, interface states.

\section{Introduction}

High permittivity (high- $\kappa$ ) dielectrics are subject of great study in order to replace $\mathrm{SiO}_{2}$ as gate dielectric in metal-oxidesemiconductor field-effect (MOS-FET) transistors in the future scales of integration [1]. $\mathrm{HfO}_{2}$ is among the most promising high- $\kappa$ dielectrics, but before these materials can replace $\mathrm{SiO}_{2}$ as gate dielectric, the nature and formation of electrically active defects existing in these materials should be known because they play an important role in device operation. Moreover, $\mathrm{HfO}_{2}$, as most of the high-k materials, when deposited in direct contact with $\mathrm{Si}$ an interfacial oxide layer few nanometers thick is formed [2]. One of the possible procedures to avoid reaction on Si surface is to grow a controlled free-defect $\mathrm{SiO}_{2}$ barrier layer between substrate and high- $\kappa$ dielectric. However, this barrier layer can lead to a reduction of the dielectric constant and, hence, to the effective capacitance of the gate dielectric stack, impeding to reach the necessary equivalent oxide thickness values [ 3 and 4].

Two basic modifications of acoustic (acoustoelectric) deeplevel transient spectroscopy (A-DLTS) were introduced after the acoustoelectric effect (AE) in semiconductor structures has been shown to be one of a useful tool for the experimental study of interface states. The surface acoustic wave (SAW) technique uses a nonlinear acoustoelectric interaction between the SAW electric field and the free carriers in an interface region which generates a transverse acoustoelectric signal (TAS) across the structure [5 and 6]. The longitudinal acoustic wave (LAW) technique uses an acoustoelectric response signal (ARS) observed at the interface of the semiconductor structure when a longitudinal acoustic wave propagates through the structure [7 and 8]. Because both TAS and ARS are very sensitive to any changes in the space charge distribution in the interface region their time development after an injection pulse has been applied to the structure (A-DLTS) and their dependence on external voltage ( $U_{a c}-U_{g}$ curves) can be used to study the interface states properties including their activation energy, cross-section and concentration and interface states distribution [8 - 10].

In this contribution the MOS structures prepared on n-type Si substrate with $\mathrm{SiO}_{2} / \mathrm{HfO}_{2}$ gate dielectric layers formed by $\mathrm{HfO}_{2}$ oxide deposited by atomic layer deposition on $\mathrm{SiO}_{2}$ oxide film prepared with nitric acid oxidation of $\mathrm{Si}$ (NAOS) are investigated by the SAW and LAW A-DLTS techniques including the ARS - gate voltage $\left(U_{a c}-U_{g}\right)$ dependences to characterize the interface states and the role of annealing treatment. The obtained results are analyzed, discussed and mutually compared.

\section{Theoretical principles}

The basic principle of an ARS creation can be explained using the idea of an acoustic wave passing through the MOS structure characterized by the particular space charge region at the interface. The acoustic wave, following the modulation of charge density by acoustic pressure $p=p_{0} \cos (\omega t-k x)$ evokes

\footnotetext{
* ${ }^{1}$ Peter Bury, ${ }^{2}$ Taketoshi Matsumoto, ${ }^{1}$ Stefan Hardon, ${ }^{1}$ Ivan Bellan, ${ }^{1}$ Marian Janek, ${ }^{2}$ Hikaru Kobayashi

${ }^{1}$ Department of Physics, Faculty of Electrical Engineering, University of Zilina, Slovakia

E-mail: peter.bury@fel.uniza.sk

${ }^{2}$ Institute of Scientific and Industrial Research, Osaka University, CREST, Japan Science and Technology Organization, Osaka, Japan
} 
the change of the potential difference that manifests as an ARS signal. The ARS produced by a MOS structure propagating by longitudinal acoustic wave can be then expressed by the relation [10 and 11]

$$
\begin{aligned}
& U_{a c}=\varphi_{s} \frac{p}{K_{s}}-\frac{Q\left(\varphi_{s}\right)}{C_{o x}} \frac{p}{K_{i}}-\frac{Q_{i t}\left(\varphi_{s}\right)}{C_{o x}} \frac{p}{K_{i}}= \\
& =U_{a c}^{o} \cos (\omega t-k x)
\end{aligned},
$$

where $\varphi_{s}$ is the surface potential, $Q_{s}$ is the semiconductor charge, $Q_{i t}$ is the trapped charge at the interface state, $C_{o x}$ is the oxide capacitance, $K_{s}$ and $K_{i}$ are the elastic moduli of the semiconductor and insulator, respectively and $p_{0}$ is the acoustic pressure amplitude.

The non-destructive SAW A-DLTS technique is based on the fact that the electric field accompanying the SAW on a piezoelectric substrate can generate the TAS that reflects any changes in the space charge distribution in the interface regions. The resulting electric field across the structure calculates dc AE effect and hf wave propagation properties and can be expressed by the relation [12]

$$
E=E_{0}+E_{1} e^{-i(\omega t-k x)},
$$

where $E_{0}$ is dc part and $E_{l}$ is the amplitude of hf part of TAS, respectively. The corresponding carrier density follows the relation

$$
n=n^{0}+n_{0}+n_{1} e^{i(\omega t-k x)},
$$

where $n^{0}$ is the carrier concentration with no applied field and $n_{0}, n_{1}$ are the $\mathrm{AE}$ term and term proportional to the amplitude of exciting wave, respectively. The transversal hf acoustoelectric signal can be then given by the relation

$$
U_{a c}=\int_{0}^{d} E_{1 y} \sin (\omega t-k x) d x .
$$

Here $d$ is the selected window width. Choosing the interaction space of the $\lambda / 2$ length [or $(2 n+1) \lambda / 2$ ] in $x$ direction, where $n$ is the natural number, the average value is not zero any more and reaches its maximum.

The principle of A-DLTS technique [6, 8 and 10] is based on the fact that the change of the amplitude of the measured ARS, $\delta U_{a c}^{0}$ and/or TAS after an injection pulse has been applied is proportional to the nonequilibrium charge at the interface and the decay time constant associated with the relaxation of the ARS or TAS amplitude is then a direct measure of the time constant associated with the relaxation processes of injected carriers. Therefore, the ARS amplitude time dependence can be written as

$$
U_{a c}^{0}(t)=U_{0}+U_{1} \exp (-t / \tau)
$$

where $U_{0}$ is the original ARS (TAS) due to the acoustoelectric interaction of acoustic wave and charge at the interface of MOS structure and $U_{1}$ represents the increase of the ARS (TAS) due to the injection pulse. The time constant characterizing the relaxation processes after applied injection pulse can be expressed for electrons by the relation

$$
\tau^{-1}=\gamma_{n} \sigma_{n} T^{2} \exp \left[-\frac{E_{a}}{k_{B} T}\right]
$$

where $\sigma_{n}$ is the capture cross section, $\gamma_{n}$ is constant, $E_{a}$ is the interface state activation energy related to the bottom of conduction band, $k_{B}$ is the Boltzmann's constant and $T$ is the thermodynamic temperature. The analysis of the time dependence of the ARS or TAS at different temperatures then allows to construct the A-DLTS spectra and following to determine the activation energy of interference states $E_{a}$ and corresponding cross section $\sigma_{n}[6-11]$.

If the ARS in the MOS structure without any interface states $\left(Q_{i t}=0\right)$ is indexed as "ideal" then the ARS amplitude can be expressed [10 - 11] in the form

$U_{a c}^{0}($ ideal $)=\left|\frac{p_{0}}{K_{s}}\right|\left(U_{g}-U_{f b}\right)\left|+\frac{Q_{s}}{C_{o x}} \frac{K_{i}-K_{s}}{K_{s} K_{i}} p_{0}\right|$,

where $U_{f b}$ is the flatband voltage. As it can be seen from Eq. (7) the ARS of ideal MOS structure is the superposition of linear term with zero at flatband voltage and term representing the contribution from the semiconductor charge $Q_{s}$. Comparing Eq.(1) and Eq.(7) the interface trapped charge can be expressed through the deviation of the ARS of real and ideal structures as

$$
Q_{i t}=S\left(U_{a c}^{0}-U_{a c}^{0}(\text { ideal })\right),
$$

where $S=C_{o x} K_{s} / p_{o}$. To determine the energy distribution of interface states, it is necessary to know the dependence of the semiconductor charge on the surface potential for an ideal MOS structure. This dependence can be obtained using Terman's model [13]. The physical meaning of the absolute values is that the ARS cannot differentiate the total charge or potential polarity.

The interface state density $D_{i t}$ can be then expressed, appearing from the $U_{a c} U_{g}$ curves for ideal and real MOS structures, by the relation

$D_{i t}\left(E_{t}\right)=\frac{1}{q}\left|\frac{d Q_{i t}}{d \varphi_{s}}\right|=\frac{1}{q}\left|S \frac{d\left(U_{a c}^{0}-U_{a c}^{0}(\text { ideal })\right)}{d \varphi_{s}}\right|$,

Eq. (5) allows to determine the modeling of the distribution of interface states from the measured ARS.

However, the leakage current represented by tunneling transport in the case of very thin oxide layers $(<10 \mathrm{~nm})$ becomes a significant problem. The tunneling current for very thin oxide layers influences the division of the applied voltage $U_{g}$ between the semiconductor and insulator layer and for the oxide layer thickness $<2 \mathrm{~nm}$ the whole applied voltage practically spreads 
across the semiconductor, especially in the range of inversion [14]. Concerning the tunneling process the transport of free charge curriers through the thin oxide layer caused by applied electric field has to be taken into account where the tunneling current following the Fowler-Nordheim mechanism [15] induces additional change of the ARS

$$
\Delta U_{a c}=A\left(U_{g}^{2}+B\right) e^{-\frac{c}{U_{g}}},
$$

where $A, B$ and $C$ are constants. The tunneling current is supposed to be dependent only on the applied voltage. The simulation of the "ideal" ARS inclusive the calculation of tunnel current contribution gives a new ideal ARS, $U_{a c}^{0}$ (tunnel). The interface charge can be then expressed using the new ideal and real ARS-voltage curves for MOS structure by the relation

$$
Q_{i t}=S \mid\left(U_{a c}^{0}-U_{a c}^{0}(\text { tunnel })\right) \mid .
$$

The calculation procedure for determination of interface states density is is then given by Eq. (9). Except the leakage current the Schottky contact on the metal-semiconductor interface can be the reason of some deviation of the measured $U_{a c}-U_{g}$ dependence, especially near the flatband. To eliminate this influence, the added capacitance of Schottky contact was included into the calculation procedure.

\section{Experimental}

The MOS structure prepared on n-type Si substrate wafers with $\mathrm{NAOS}-\mathrm{SiO}_{2} / \mathrm{HfO}_{2}$ gate dielectric layer was formed by $5 \mathrm{~nm}$ $\mathrm{HfO}_{2}$ oxide deposited by atomic layer deposition on $0.6 \mathrm{~nm}$ NAOS-SiO ${ }_{2}$ oxide film prepared in $\sim 100 \% \mathrm{HNO}_{3}$ vapor [16]. The set of investigated MOS capacitors contained steh original MOS structures (A1, A2) and structures annealed in $\mathrm{N}_{2}$ atmosphere at $200(\mathrm{~A} 3, \mathrm{~A} 4), 300(\mathrm{~A} 5, \mathrm{~A} 6)$ and $400{ }^{\circ} \mathrm{C}(\mathrm{A} 7, \mathrm{~A} 8)$ for 10 min to stabilize the structure and decrease the leakage current density. After annealing procedure, aluminum dot electrodes were evaporated. The samples size was $2 \times 2 \mathrm{~cm}^{2}$ and electrodes were evaporated with diameter $2.5,1.5$ and $1.0 \mathrm{~mm}$. The investigated samples list and obtained experimental results are summarized in Table 1. It should be noted that couples of samples A1-A2, A3-A4, A5-A6 and A7-A8 represent the same structures but prepared for LAW or SAW techniques, respectively.

The block diagram of the experimental setup for A-DLTS is illustrated in Fig. 1. The computer was used to trigger the apparatus, to generate excitation bias pulses as well as to record and evaluate the isothermal transients of the ARS and/or TAS. The LAW of frequency $13.2 \mathrm{MHz}$ and SAW of frequency 10 $\mathrm{MHz}$ were generated using $\mathrm{LiNbO}_{3}$ transducer and interdigital transducer evaporated on $\mathrm{LiNbO}_{3}$ delay line, respectively in the arrangement illustrated in the A- and B-detail of Fig. 1.
The acoustoelectric signals produced by MOS structure were after detection in the Box-car Averager recorded and stored by computer. The quiescent bias voltage pulses of $200 \mathrm{~ms}$ with filling traps completely were applied to the MOS structures. The ARS and TAS were monitored as a function of temperature and peaks with maxima of the temperature for which the emission rate was the same as the adjusted sample rate were observed in A-DLTS spectra [8 - 10]. The A-DLTS experimental results were at temperatures varying between 400 and $77 \mathrm{~K}$, with the samples cooled in nitrogen cryostat.

To determine the interface states density the $U_{a c}-U_{g}$ measurements were performed using the same acoustic equipment as for A-DLTS with the difference of bias source. Whereas for the A-DLTS the voltage source provided with bias voltage pulses, for the measurement of $U_{a c}-U_{g}$ curves the voltage source provided with both the linear increase (decrease) of gate bias and the variation of increasing (decreasing) rate. Current voltage (I-V) and capacitance voltage $(C-V)$ characteristics were observed using FLUKE PM 6306, programmable automatic RLC meter.

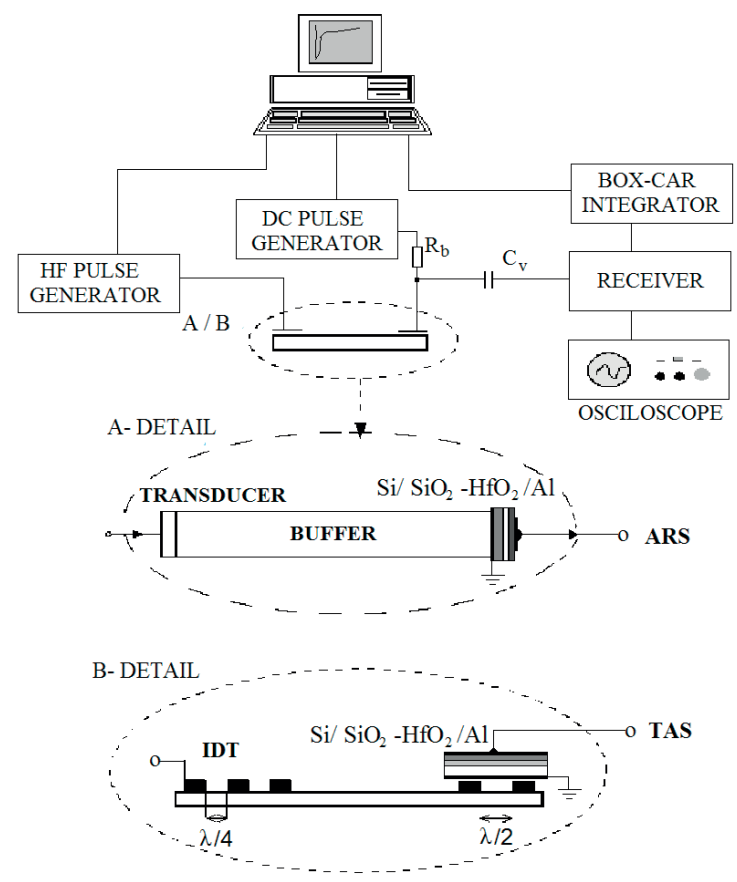

Fig. 1 Block diagram of the experimental setup for A-DLTS. The sample configuration for LAW technique is in A-detail and For SAW in B-detail

\section{Results and discussion}

Figure 2 shows the representative SAW A-DLTS spectrum of original $\mathrm{Si} / \mathrm{SiO}_{2} / \mathrm{HfO}_{2}$ structure and calculated Arrhenius plot inside. The illustrated spectrum that was observed with pulse voltage $\Delta U g=+4.0 \mathrm{~V}(U g=-1 \mathrm{~V})$, similarly as other samples, 
contains one evident peak with some lateral structure that gradually decreases from the original structure A2 to the sample A8 annealed at $400^{\circ} \mathrm{C}$. Except for this "cleaning process" there was observed also some shift of the activation energy in regard to the original structure.

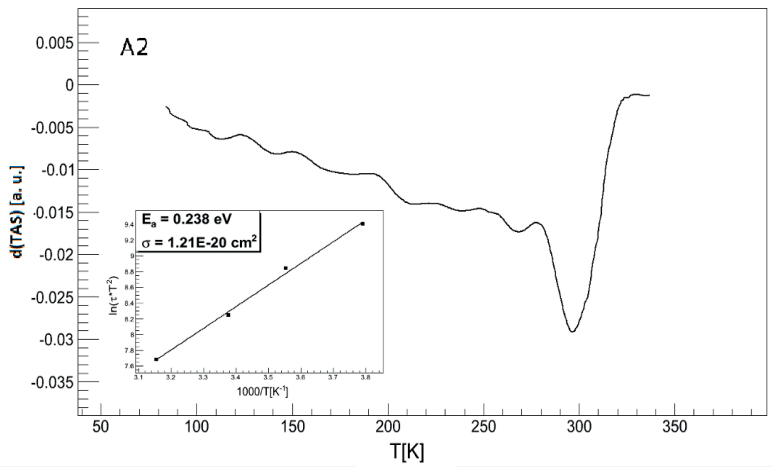

Fig. $2 \mathrm{SAW}$ A-DLTS spectrum of $\mathrm{Si} / \mathrm{SiO} / 2 \mathrm{HfO}_{2}$ structure (A2) and calculated Arrhenius plot (inside)

Figure 3 presents a representative A-DLTS spectrum of original structure A1 obtained using LAW technique and calculated Arrhenius plot (inside). The illustrated spectrum that was observed with pulse voltage $\Delta U_{g}=+3.0 \mathrm{~V}(U g=0 \mathrm{~V})$ contains one evident peak with some lateral structure that also gradually decreases from the original structure A1 to the sample A7 annealed at $400^{\circ} \mathrm{C}$ [11]. Except for this reducing process there was observed also some shift of the activation energy in regard of the original structure. Therefore, the interface quality using the interfacial $\mathrm{SiO}_{2}$ layer seems not to be influenced by the annealing process markedly.

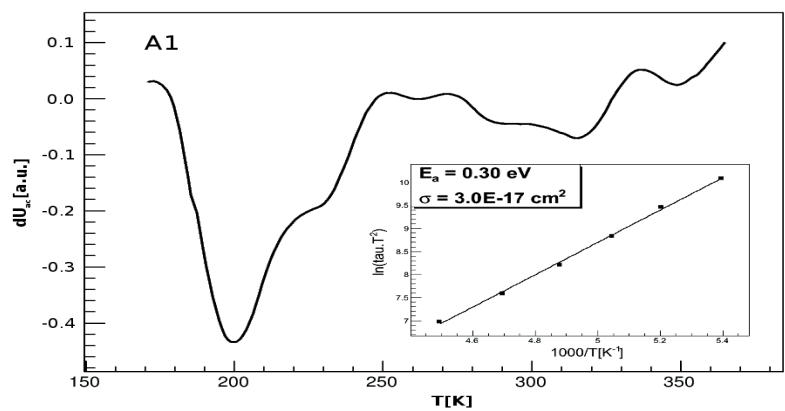

Fig. $3 \mathrm{LAW}$ A-DLTS spectrum of $\mathrm{Si} / \mathrm{SiO} \mathrm{O}_{2} \mathrm{HfO}_{2}$ structure

(A1 sample) and calculated Arrhenius plot (inside)

The results of both TAW and SAW A-DLTS are summarized in Table 1. The activation energies and corresponding cross sections of interface states were determined from the A-DLTS spectra, observed from the ARS and/or TAS amplitude transients measured at different temperatures.

The Electron Spin Resonance data [2] indicated that the basic defects observed at the $\mathrm{Si} / \mathrm{HfO}_{2}$ interface were identical to those found in conventional thermally oxidized silicon. However, the tunneling process from inner layer interface states is possible, too. Both kinds of interface states detected by SAW and/or LAW technique were already observed [3 and 17]. Interface states observed on $\mathrm{Si} / \mathrm{SiO}_{2} / \mathrm{HfO}_{2}$ structures are usually attributed to isolated Si dangling bonds with which no atoms in the oxide layer interact and/or attributed to Si dangling bonds interacting weakly with an oxygen or $\mathrm{Si}$ atom in the oxide layer, respectively. The difference between SAW and LAW A-DLTS results can be caused by the different mechanism of SAW and LAW interaction with charge at the interface region, as well as the shift of applied voltage $U_{g}$ in the case of SAW technique due to the dc transversal acoustoelectric voltage (TAV) produced by structure [5 and 12].

Summarization of the investigated $\mathrm{Al} / \mathrm{NAOS}-\mathrm{SiO}_{2}-\mathrm{HfO}_{2} / \mathrm{Si}$ MOS structures, activation energies and cross-sections provided by LAW and SAW A-DLTS

\begin{tabular}{|c|c|c|c|c|c|}
\hline \multirow{2}{*}{} & \multirow{2}{*}{ Annealing process } & \multicolumn{2}{|c|}{$E_{a}[\mathrm{eV}]$} & \multicolumn{2}{c|}{$\sigma\left[\mathrm{cm}^{2}\right]$} \\
\cline { 3 - 6 } & Without annealing & LAW & SAW & LAW & SAW \\
\cline { 3 - 6 } A1 & Without annealing & 0.30 & & $3.0 \times 10^{-17}$ & $1.2 \times 10^{-20}$ \\
\hline A2 & $\mathrm{N}_{2}$ at $200^{\circ} \mathrm{C}$ for $10 \mathrm{~min}$ & 0.28 & & $8.4 \times 10^{-17}$ & $6.6 \times 10^{-22}$ \\
\hline A3 & $\mathrm{N}_{2}$ at $200^{\circ} \mathrm{C}$ for $10 \mathrm{~min}$ & & 0.17 & & $9.8 \times 10^{-21}$ \\
\hline A4 & $\mathrm{N}_{2}$ at $300^{\circ} \mathrm{C}$ for $10 \mathrm{~min}$ & 0.37 & & $4.0 \times 10^{-16}$ & \\
\hline A5 & $\mathrm{N}_{2}$ at $300^{\circ} \mathrm{C}$ for $10 \mathrm{~min}$ & & 0.20 & & $1.2 \times 10^{-21}$ \\
\hline A6 & $\mathrm{N}_{2}$ at $400^{\circ} \mathrm{C}$ for $10 \mathrm{~min}$ & 0.33 & & $6.1 \times 10^{-17}$ & \\
\hline A7 & $\mathrm{N}_{2}$ at $400^{\circ} \mathrm{C}$ for $10 \mathrm{~min}$ & & 0.19 & & \\
\hline A8 & & & & & \\
\hline
\end{tabular}




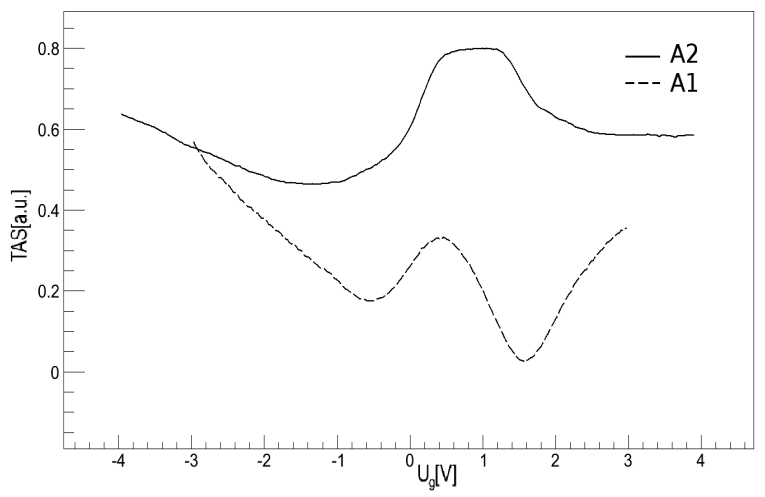

Fig. 4 Dependence of the TAS and ARS on gate voltage Ug for original structures, $A 1$ and $A 2$

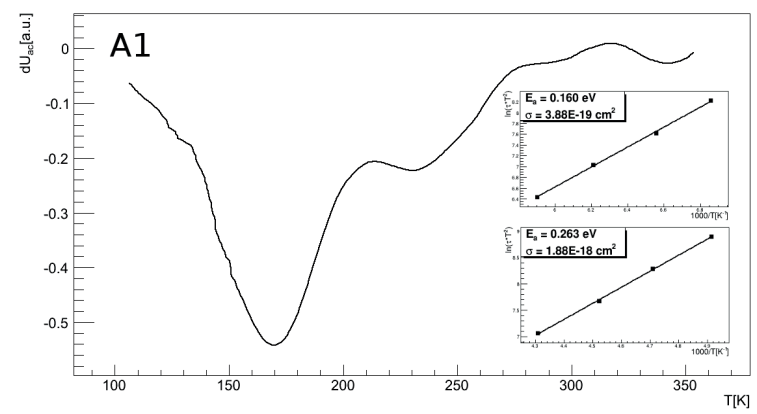

Fig. $5 \mathrm{LAW}$ A-DLTS spectrum of $\mathrm{Si} / \mathrm{SiO} / \mathrm{HfO}_{2}$ structure (A1 sample) and calculated Arrhenius plots (inside) obtained for $\Delta U_{g}=+5.0 \mathrm{~V}$,

$$
U_{g}=-2.0 \mathrm{~V}
$$

Figure 4 presents the dependences of the ARS as well as TAS on the gate voltage for original structures $\mathrm{A} 1$ and $\mathrm{A} 2$, respectively. Besides, the LAW technique can identify the role of interfacial $\mathrm{SiO}_{2}$ layer (interface $\mathrm{SiO}_{2} / \mathrm{HfO}_{2}$ ) and corresponding tunneling, the SAW technique is not so sensitive.

However, using pulse voltage $\Delta U_{g}=+5.0 \mathrm{~V}\left(U_{g}=-2.0 \mathrm{~V}\right)$, similar as in the case of SAW spectra we found as a certain shift of A-DLTS spectra obtained for only positive voltage $\left(\Delta U_{g}=+3.0 \mathrm{~V}\right.$, $\left.U_{g}=0 \mathrm{~V}\right)[11]$ as also some highlighting of another peak at lower temperatures $(\sim 170 \mathrm{~K})$ corresponding to the interface state with activation energy $\sim 0.12-0.16 \mathrm{eV}$ (see Fig. 5 ). This fact indicates as the typical feature of interface states moreover the role of $\mathrm{HfO}_{2} /$ $\mathrm{SiO}_{2} /$ interlayer (IL).

The energy diagrams of the MOS structures under accumulation and inversion are illustrated in Fig. 6. We also assume that defects exist also at the $\mathrm{HfO}_{2} / \mathrm{SiO}_{2} \mathrm{IL}$ as well as at $\mathrm{Si} / \mathrm{SiO}_{2}$ interface. A-DLTS measurements consist of applying accumulation pulses to fill the interface states in the upper half of the semiconductor band gap followed after reverse voltage in which the interface states emit electrons to the conduction band yielding the ARS or TAS transients that are conveniently recorded and processed to obtain the A-DLTS spectra. If the $\mathrm{SiO}_{2}$ film is thin enough, tunneling between the semiconductor and the IL may occur. At accumulation,

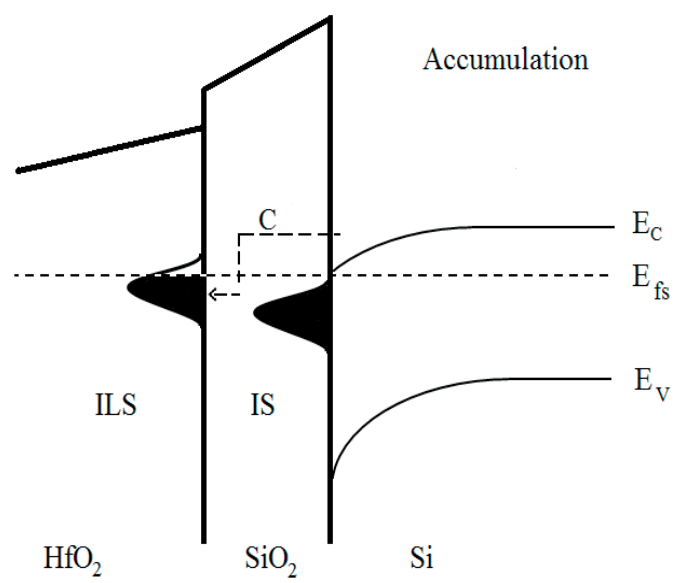

(a)

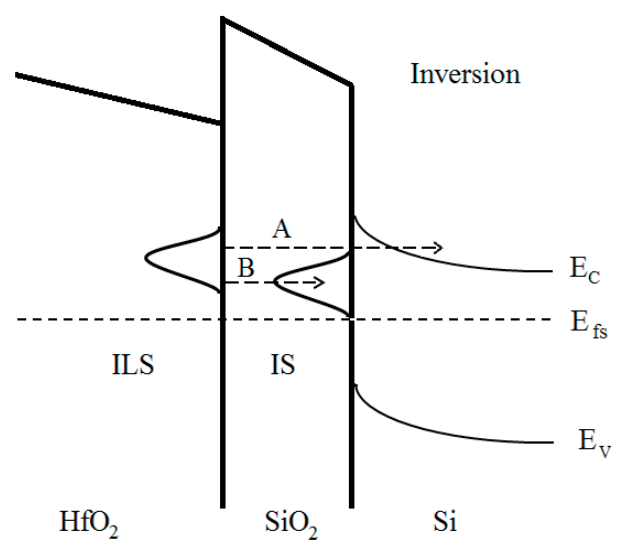

(b)

Fig. 6 Energy band diagram of $\mathrm{Si} / \mathrm{SiO} / \mathrm{HfO}_{2}$ structure at accumulation (a) and inversion (b)

capturing electrons coming from the semiconductor band by direct tunneling fills IL states. When the reverse pulse is applied, these defects emit the captured electrons to the semiconductor band. The emission process may occur in two different ways: IL states with energies above the silicon conduction band emit electrons by direct tunneling (A). On the other hand, for energies ranging from the Fermi level to the semiconductor conduction band tunneling between the IL states and the interface states (B) and following to the conduction band can occur. Electrons emitted according the (B) sequence increase the acoustoelectric transient, obtaining an 
apparent increase in the measured interfacial state densities. Since all these mechanisms are tunneling assisted the thinner the silicon dioxide films the higher their probability. In our experiment, the $\mathrm{SiO}_{2}$ layer thickness was $0.6 \mathrm{~nm}$.

Moreover, an increase in the filling electric field in Fig. 6(a) (higher bias in the accumulation regime) causes a larger number of IL filled traps $(\mathrm{C})$. Then, when biasing the sample in the inversion regime, a higher number of IL traps can contribute to the ARS or TAS transients by direct tunneling. This result agrees with results shown in [3]. On the contrary, variations in the inversion bias should not change the total filled traps, and the emitted charge from the IL traps does not change - significantly.

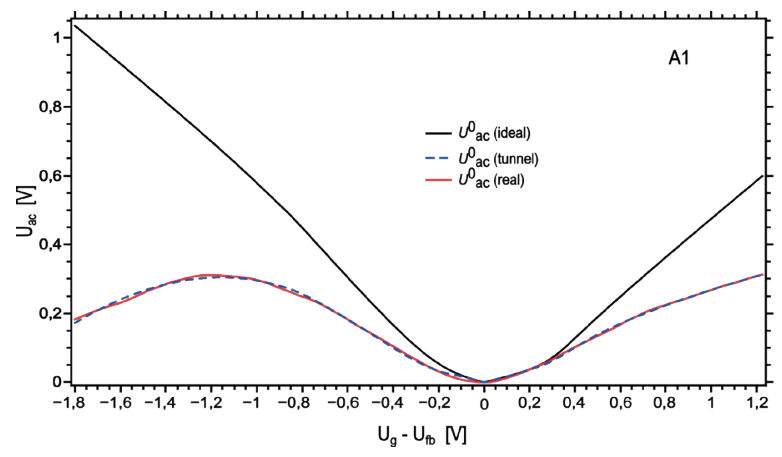

Fig. 7 Theoretical $U_{a c}-U_{g}$ characteristics of "ideal" $\mathrm{Si} / \mathrm{SiO} / \mathrm{HfO}_{2}$ structure without any interface states (thick line) and "ideal" structure with tunneling process (dashed line) compared with real $U_{a c}-U_{g}$ curve (red line)

Figure 7 shows the measured ARS dependence on gate voltage, $U_{a c}-U_{g}$ curve for sample A1 (Fig. 4) including the theoretical (ideal) $U_{a c}-U_{g}$ characteristics for the $\mathrm{Si} / \mathrm{SiO}_{2} / \mathrm{HfO}_{2} / \mathrm{Al}$ MOS structure without any interface states as well as simulated characteristics for the same structure comprehensive of tunneling process. Using these real and theoretical characteristics the density of interface states could be determined (Fig. 8). We can see, in the first place, that our theoretical calculation of ideal $U_{a c}-U_{g}$ curve very well coincides with real one and, secondly, that the density of interface states course corresponds to the presence of interface states with energies around $\sim 0.2-0.3 \mathrm{eV}$ detected also by A-DLTS (Table 1). The average values of density of interface states calculated using the correct value of donor concentration, $\mathrm{N}_{\mathrm{D}}=4.7 \times 10^{21} \mathrm{~m}^{-3}$ and considering the Schottky contact, changed from $2.64 \times 10^{12} \mathrm{eV}^{-1} \mathrm{~cm}^{-2}$ (A1) up to $1.05_{x} 10^{12}$ $\mathrm{eV}^{-1} \mathrm{~cm}^{-2}$ (A7).

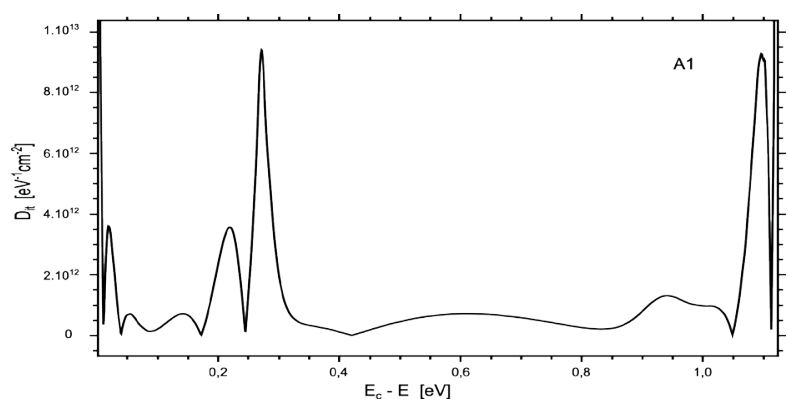

Fig. 8 Distribution of interface states calculated from $U_{a c}^{0} U_{g}$ characteristics for original structure (A1)

The reduction of the leakage current densities for annealed structures is evident only for the gate voltage close to zero (from $-0.3 \mathrm{~V}$ to $+0.3 \mathrm{~V}$ ) decreasing from sample A1 gradually to sample A7 [11]. The behavior of $I-V$ curves corresponds to theoretical predictions for such structures [14] but except for the mentioned interval it is almost identical for both untreated and treated structures, although there were significant differences in $C-V$ characteristics. The reason of such behavior could be caused by two kinds of interface states, at both $\mathrm{SiO}_{2} / \mathrm{Si}$ and $\mathrm{HfO}_{2} / \mathrm{SiO}_{2}$ interfaces. Then the tunneling process through the $\mathrm{SiO}_{2}$ interfacial layer at accumulation and/or depletion can lower the influence of the annealing treatment [ 3 and 11$]$

\section{Conclusion}

MOS structures with $\mathrm{HfO}_{2}$ oxide layer formed on n-type silicon with interfacial $\mathrm{SiO}_{2}$ layer prepared by NAOS method and annealed in $\mathrm{N}_{2}$ at various temperatures were studied by LAW and SAW acoustic spectroscopy. The main interface states with activation energy of $0.17-0.23 \mathrm{eV}$, observed by SAW A-DLTS are quite different from those found by LAW A-DLTS (0.27-0.37 $\mathrm{eV})$ typical for dangling bonds type defects and identical to those in $\mathrm{Si} / \mathrm{SiO}_{2}$ interface. These differences can be caused by both the different mechanism of SAW and LAW interaction with charge at the interface region and due to the dc transversal acoustoelectric voltage produced by structure. Except for this, a post-deposition annealing at 200,300 , and mainly at $400^{\circ} \mathrm{C}$ leads to the reduction of interface state density, hysteresis effect as well, but only insignificantly leakage current density. The lowering influence on the characteristics of annealed MOS structures with NAOS-SiO $/$ $\mathrm{HfO}_{2}$ gate dielectric layers can indicate the stabilization role of the NAOS-SiO interfacial layer. However, the next reduction should be realized by appropriate modification of structure processing steps. An observed shift of the interface state activation energies and some "cleaning" process observed in annealed structures can be explained by reduction of weak Si dangling bonds with surrounding atoms. 


\section{Acknowledgements}

The authors would like to thank to Mr. F. Černobila for technical assistance and Assoc. prof. I. Jamnický for some remarks. This work was financially supported by the R\&D operational program Centrum of excellence of power electronics systems and materials for their components II. No. OPVaV2009/2.1/02-SORO, ITMS 26220120046 funded by the European Community.

\section{References}

[1] LOCQUET, J.P., MARChIORI, C., SOUSA, M., FOMPEYRINE, J., SEO, J. W.: J. App. Phys. 100, 2006, 051610

[2] STESMANS, A., AFANAS ' EV, V. V.: Appl. Phys. Lett. 82, 2003, 4074

[3] CASTAN, H., DUENAS, S., GARCIA, H., GOMEZ, A., BAILON, L., TOLEDANO-LUQUE, M., DEL PRADO, A., MARTIL, I., GONZALEZ-DIAZ, G.: J. Appl. Phys. 107, 2010, 114104

[4] HEE-WOOK YOU, WON-JU CHO: Appl. Phys. Lett. 96, (2010) 093506

[5] ABATE, A., MAN, K. J., OSTROVSKIJ, I. V., DAS, P.: Solid State Electronics 36 1993, 697

[6] BURY, P., JAMNICKY, I., RAMPTON, V. W.: Phys. B 94, 1999, 263

[7] BURY, P., JAMNICKY, DURCEK, J.: Phys. Stat. Sol. A 151, 1991, 126

[8] BURY, P., JAMNICKY: Acta Phys. Slovaca 46, 1996, 693

[9] HOCKICKO, P., BURY, P., SIDOR, P., JURECKA, S., JAMNICKY, I.: Communications - Scientific Letters of the University of Zilina, 12, 2010, 44

[10] HOCKICKO , P., BURY, P., SIDOR, P., KOBAYASHI, H., TAKAHASHI, M., YANASE, T.: Cent. Eur. J. Phys. 9, 2011,242

[11] BURY, P., MATSUMOTO, T., BELLAN, I., JANEf́K, M., KOBAYASHI, H.: Applied Surface Sciences 269, 2013, 50

[12] FRITZ, I. J.: J. Appl. Phys. 52, 1981, 6749

[13] NICOLLIAN, E. M., BREWS, J. R.: MOS (Metal Oxide Semiconductor), Physics and Technology, New York : Wiley, 1982.

[14] RACKO, J., VALENT, P., BENKO, P., DONOVAL, D., HARMATHA, L., PINTES, P., BREZA, J.: Solid State Electronics 52 , 2008, 1755.

[15] WEINBERG, Z. A.: Solid State Electronics 20, 1977, 11.

[16] ASUHA, A., YUSA. T., MAIDA, O ., KOBAYASHI, H.: Appl. Phys. Lett. 80, 2002, 4175.

[17] MASSON, P., AUTRAN, J.-L., HOUSSA, M.: App. Phys. Lett. 81, 2002, 3392. 